\title{
Apuntes sobre el enfoque de la complejidad y su aplicación en la salud
}

\section{Notes on the approach to complexity and its application in the field of healthcare}

\author{
José Antonio Betancourt Betancourt ${ }^{\text {I }}$; Rina M. Ramis Andalia ${ }^{\text {II }}$ \\ IMáster en Salud Pública. Epidemiólogo. Universidad de Ciencias Médicas "Carlos J. \\ Finlay". Camagüey, Cuba. \\ IIMáster en Salud Pública. Epidemióloga. Investigador Auxiliar. Escuela Nacional de \\ Salud Pública. La Habana, Cuba.
}

\section{RESUMEN}

La medicina en su esquema metodológico tradicional al igual que otras tantas disciplinas, ha tenido un enfoque basado en la mecánica de Descartes y Newton, por lo que en muchas ocasiones mantiene el esquema tradicional de causa-efecto basado en modelos de ecuaciones lineales, donde todo funciona como una maquinaria de forma predecible. En el presente trabajo se argumenta como el enfoque de los sistemas complejos fomenta una forma de actuación emergente, holista, transdisciplinar que integra técnicas y conocimientos de diversos campos. El artículo destaca como este enfoque ofrece las herramientas adecuadas para implementar los nuevos modelos necesarios. Se detallan algunas propiedades de los sistemas complejos con el fin de divulgar este enfoque en el sector de la salud. Para realizar este artículo se consultaron más de 50 fuentes bibliográficas y se realizaron entrevistas y consultas a especialistas. La teoría de la complejidad, proporciona al médico una gran capacidad de generar nuevas soluciones integradas a través de las conexiones históricas y sociales de cada localidad, para un conocimiento expandido; capacidad para el debate, la discusión, adaptación a los cambios y capacidad de desarrollar nuevas metas y estrategias. 
Palabras clave: Sistemas complejos de salud, salud y complejidad.

\begin{abstract}
Like many other disciplines, the approach of the traditional methodological scheme of medicine has been based on Descartes and Newton's system, therefore medicine keeps in many occasions the traditional cause-effect pattern based on linear equation models where everything operates in a predictable way like a piece of machinery. The present paper substantiated how the complex system approach encourages emerging, holistic, multidisciplinary way of acting that incorporates techniques and knowledge from several fields. The article stresses how this approach provides the suitable tools to implement new required models. Some properties of the complex systems were described in order to disseminate this type of approach in the healthcare sector. To write this article, a literature review was made, which comprised 50 sources and several interviews to specialists. The complexity theory provides the physician with great capacities of generating new comprehensive solutions through the historical and social relationships existing in each place, giving rise to broader knowledge, greater capacity for debate, discussion, adaptation to change and increased capabilities to develop new targets and strategies.
\end{abstract}

Key words: Complex health systems, health and complexity.

\title{
INTRODUCCIÓN
}

La medicina en su esquema metodológico tradicional al igual que otras tantas disciplinas, ha tenido un enfoque basado en la mecánica de Descartes y Newton, por lo que en muchas ocasiones mantiene el esquema tradicional de causa-efecto basado en modelos de ecuaciones lineales, donde todo funciona como una maquinaria de forma predecible. ${ }^{1}$ Sin embargo, hoy se manifiestan otras situaciones como que: a. El hombre trabaja con modelos de la realidad y muchos de los que tradicionalmente se están usando comienzan a tener contradicciones. $b$. No existen suficientes modelos que expliquen satisfactoriamente determinados fenómenos dentro de las formas de movimiento biológico y social. c. Existe un desarrollo apreciable de las matemáticas y en general de las ciencias básicas, que permite plantear modelos matemáticos más cercanos al comportamiento real del sistema. d. Hay un vertiginoso desarrollo de las técnicas de información y comunicación.

Para dar respuestas a este problema existe un nuevo enfoque y este es el enfoque de los sistemas complejos. ${ }^{2}$ Estos se distinguen de los simples, por la emergencia de determinados comportamientos y patrones resultantes de relaciones entre los elementos que los integran. Los sistemas complejos tienen propiedades que lo caracterizan, ${ }^{3-8}$ algunas de estas son:

a. Relaciones no-lineales entre elementos del sistema. b. Estructura en red, ramificada. c. Poca predicción a largo plazo. d. Existencia de fluctuaciones 
(internas) y de perturbaciones (externas). e. Las fronteras del sistema son borrosas y dinámicas. f. Evolución temporal acotada (atractores). g. Procesos de retroalimentación. h. El sistema tiene una historia y es único e irrepetible en el tiempo (singularidad). i. Autoorganización.

A continuación se relacionan estas propiedades en el trabajo de la salud con el fin de que se conozcan elementos esenciales del enfoque de la complejidad para el personal de esta rama.

\section{RELACIONES NO-LINEALES ENTRE ELEMENTOS DEL SISTEMA}

Los sistemas complejos están formados por elementos que interactúan de forma dinámica entre si de forma no lineal. La información contenida en el sistema en conjunto es superior a la suma de la información de cada parte analizada individualmente. La linealidad de un sistema permite a los investigadores hacer ciertas suposiciones matemáticas y aproximaciones, lo que admite un cálculo más sencillo de los resultados. Desde que los sistemas no lineales no son iguales a la suma de sus partes, usualmente son difíciles (o imposibles) de modelar, y sus comportamientos con respecto a una variable dada (por ejemplo, el tiempo) es extremadamente difícil de predecir.

El enfoque no lineal prepara al médico a esperar lo inesperado, a tener en cuenta que en muchas ocasiones los resultados son impredecibles, ya que la persona con una dolencia tiene una relación dinámica con otros sistemas, que en muchos casos no se conocen (ambiente, familia, trabajo, entre otros), estas influencias externas (llamadas perturbaciones) producen cambios (oscilaciones) que pueden influir en su estado de salud en uno u otro sentido. De manera que no siempre los tratamientos y manejos tradicionalmente adecuados conducen a los resultados esperados ${ }^{9}$ y el médico debe anticiparse a estas situaciones. Es común que los pacientes con diabetes ${ }^{10}$ y con hipertensión arterial no respondan al tratamiento y manejo de la forma esperada. ${ }^{11,12}$

La medicina se ha analizado desde el enfoque de la complejidad, argumentando la presencia de incertidumbre, dinámica no-lineal y causalidad compleja. ${ }^{13}$ Para enfrentar la incertidumbre y para elevar la capacidad de tomar iniciativas, el profesional y el estudiante de la salud deben ser entrenados con guías y simulaciones ${ }^{14}$ deben participar activamente en proyectos de investigación para resolver problemas locales y aprender a recoger y utilizar observaciones sistemáticas del trabajo cotidiano en bases de datos adecuadas. ${ }^{4}$

En el enfoque epidemiológico prevalece el análisis de relaciones causa-efecto con utilización de análisis lineales enfocados en buscar variables dependientes e independientes y no se utilizan sistemáticamente otras herramientas propias del enfoque de la complejidad, que midan de forma realista las interrelaciones entre elementos del sistema que se pueden encontrar al utilizar análisis matemáticos no lineales y simulaciones. ${ }^{3}$

\section{ESTRUCTURA EN RED, RAMIFICADA}

Los sistemas complejos al interactuar entre si lo hacen formando una red ramificada. En salud es esencial evaluar la interacción de los individuos y su entorno, que incluye infinidad de variables como pueden ser ambiente, clima, entorno laboral, familiar, político y otros. Una red social es una estructura que se puede representar en forma de uno o varios grafos en el cual los nodos representan 
individuos (a veces denominados actores) y las aristas, relaciones entre ellos. Las relaciones pueden ser de distinto tipo, como intercambios financieros, amistad, relaciones sexuales o rutas aéreas. También es el medio de interacción de distintas personas como por ejemplo juegos en línea, charlas, foros, entre otros. Para el reconocimiento de formas y toma de decisiones, se manejan las imprecisiones e incertidumbres que aparecen cuando se trata de resolver problemas relacionados con el mundo real con un nuevo tipo de análisis basado en este principio de estructura de redes, son las llamadas redes neuronales que ofrecen soluciones robustas y de fácil implementación.

Estas redes neuronales son sistemas de computación compuestos por un gran número de elementos de procesos simples, denominados nodos o neuronas, que procesan información por medio de su estado dinámico como respuesta a entradas externas. Las conexiones sirven para transmitir las salidas de unos nodos a las entradas de otros. El funcionamiento de un nodo es similar al de las neuronas biológicas del cerebro, donde la información memorizada está relacionada con los valores sinápticos de las conexiones entre las neuronas. Estos modelos son excelentes para evaluar encuestas, para realizar predicciones de series de tiempo y para la evaluación de influencias multivariadas tanto cualitativas como cuantitativas sobre determinado aspecto, por lo que constituye una excelente arma para el trabajo y la investigación médica. Existen modernos análisis matemáticos basados en redes, que permiten hacer simulaciones de la realidad y predicciones a corto plazo que apoyan de forma científica la toma de decisiones. ${ }^{15}$ [Koopman JS. Infection transmission science and models. 2005;58(6):S3-8].

Muchos sistemas biológicos, sociales o de comunicación se pueden describir adecuadamente a través de redes complejas cuyos nodos representan individuos $u$ organizaciones, y los enlaces simbolizan las interacciones entre ellos. Como ejemplos están las conexiones neuronales en algunos gusanos, el patrón de difusión de una epidemia, la estructura de una red de transmisión eléctrica, la navegación a través Internet, las proteínas en una célula humana, los patrones lingüísticos, las redes de colaboración social, las relaciones entre especies de un ecosistema y muchos más.

El cuerpo humano posee redes ramificadas para las funciones fisiológicas, por ejemplo, los grandes vasos sanguíneos hasta sus capilares, la red de neuronas con sus dendritas y sinapsis y el sistema linfático. En el tratamiento con medicamentos y otros en el caso de la acupuntura, el estímulo se transmite a través de una red de este tipo.

Las redes permiten la interconexión entre elementos del sistema. En la organización del trabajo de salud se han podido detectar fallas en la comunicación entre sistemas que se desconectan temporalmente y afectan los servicios; la evaluación de redes brinda una percepción global útil para reconocer las fallas y corregirlas.

\section{POCA PREDICCIÓN A LARGO PLAZO}

Los sistemas complejos poseen un comportamiento impredecible. Solo se puede prever su evolución futura hasta ciertos límites, siempre suponiendo un margen de error muy creciente con el tiempo.

Para realizar predicciones más o menos precisas de sistemas complejos se han de usar métodos matemáticos de avanzada según el tipo de dinámica que lo 
caracterice (redes Bayesianas y de Markov, lógica difusa, modelos epidemiológicos SIR, modelos de redes neuronales, modelos $\mathrm{GARCH}$, simulaciones y otros).

Los sistemas complejos pueden presentar multiplicidad en sus estados y alternativas (multifurcaciones). En ocasiones son tantos los estados que el sistema se encuentra en caos, es decir, posee infinitas posibilidades de movimiento. Esto dificulta el proceso de predicción. Muchas de las predicciones basadas en series de tiempo de enfermedades que se han estudiado no pueden ser realizadas con modelos lineales, ya que no corresponden al comportamiento real que tienen estas series como sistema, ${ }^{16}$ se deben adecuar los modelos a los sistemas y estos deben responder mejor a la realidad, pero aún así, las predicciones que se pueden realizar son a corto plazo. En la provincia de Camagüey se han estudiado algunas series de tiempo (mortalidad infantil, infección respiratoria aguda, enfermedad diarreica aguda, varicela, entre otras), que se han caracterizado por un comportamiento no lineal. ${ }^{17}$

\section{EXISTENCIA DE FLUCTUACIONES (INTERNAS) Y PERTURBACIONES (EXTERNAS)}

Un sistema adaptativo es un sistema autoorganizado, capaz de reaccionar a estímulos externos respondiendo ante cualquier situación que amenace su estabilidad como sistema. Experimenta así, fluctuaciones. Esto tiene un límite, naturalmente. Se dice que el sistema se acomoda en un estado y que cuando es apartado de tal estado tiende a hacer todos los esfuerzos posibles para regresar a la situación acomodada. Esto ocurre por ejemplo con el cuerpo humano que lucha constantemente para mantener una misma temperatura corporal, o las estrellas cuya estructura se acomoda para mantener siempre una luminosidad casi constante.

La fluctuación es el cambio en la magnitud de alguna cantidad física a lo largo del tiempo, con respecto a su valor normal o promedio. Las fluctuaciones son oscilaciones internas en el comportamiento del sistema que pueden provocar cambios de dinámica. Hay pacientes, con disímiles resultados debidos a características internas que el médico debe tener en cuenta en su análisis inicial. Es posible que se produzcan inesperadas recidivas, hecho al que el médico debe anticiparse para producir las perturbaciones terapéuticas necesarias y preparar otras medidas de manejo para la persona dentro de su contexto. La edad, la existencia de enfermedades crónicas, el genotipo y otros son contextos individuales que provocan fluctuaciones.

\section{LAS FRONTERAS DEL SISTEMA SON BORROSAS Y DINÁMICAS}

En la lógica clásica una proposición solo admite dos valores: verdadero o falso. La lógica difusa se caracteriza por querer cuantificar esta incertidumbre. La lógica difusa o lógica Fuzzy, es utilizada en algunos sistemas expertos y en otras aplicaciones de inteligencia artificial en la que las variables pueden tener varios niveles de verdad o falsedad representados por rangos de valores entre el 1 (verdadero) y el 0 (falso). Con la lógica Fuzzy, el resultado de una operación se puede expresar como una posibilidad y no necesariamente como una certeza. Por ejemplo, la clasificación de la hipertensión arterial puede ser variada por criterios de expertos basados en la experiencia local y puede adoptar valores tales como, morbosamente altos, moderadamente altos, normales, bajos, dado por el grado de pertenencia de los valores para uno u otro conjunto que estos consideren. 
Es usual que la descripción de las enfermedades se realice mediante términos lingüísticos que en general son vagos e imprecisos, sin embargo cuando la información se brinda como una posibilidad y no como una certeza entonces la información es más exacta. El análisis epidemiológico de cualquier enfermedad involucra varios niveles de incertidumbre ${ }^{18}$ dado que las enfermedades pueden manifestarse de forma diferente en dependencia del entorno, de la vulnerabilidad del ente susceptible y la virulencia del agente patógeno, este hecho se utiliza en modelos matemáticos que ubican diferentes variables según su grado posible de pertenencia a un conjunto o al otro. Para el análisis de modelos epidémicos, los modelos de Bayes o de Markov, se basan en probabilidades, sin embargo, los modelos que utilizan el principio de la lógica difusa se basa en posibilidades, esta información está documentada en experiencias para evaluar campañas de vacunaciones. ${ }^{19}$

\section{EVOLUCIÓN TEMPORAL ACOTADA (ATRACTORES)}

Todo sistema complejo emerge a partir de sus partes y fluctúa hasta quedar fuertemente estabilizado en un atractor. Esto lo logra con la aparición de toda una serie de retroalimentaciones (o realimentaciones) positivas y negativas que atenúan cualquier modificación provocada por un accidente externo. Se puede afirmar que el sistema reacciona ante agresiones externas que pretenden modificar su estructura. Tal capacidad solo es posible mantenerla sin ayuda externa mediante un aporte constante de energía. El atractor extraño es característico de los fenómenos de comportamiento caótico. Tiene formas muy variadas con trayectorias impredecibles localmente, pero circunscritas en un subespacio, presentándose así, la llamada estabilidad global con inestabilidad local.

En el organismo humano se producen constantemente bifurcaciones que mantienen o no el estado normal de salud, en ese recorrido, o bien se está al borde del caos o dentro de este. Las series de tiempo formadas por salidas fisiológicas del cuerpo humano tienen un comportamiento dinámico no lineal que en ocasiones se reflejan como un atractor extraño, es decir, en un comportamiento especial acotado, De forma metafórica se les ha llamado atractores negativos al alcoholismo, tabaquismo, y otros hábitos de vida que influyen en la salud del individuo y que están profundamente enraizados y son resistentes a los cambios, así que una manera de enfrentarlos, sería encontrar un atractor positivo para cada paciente en su contexto.

\section{PROCESOS DE RETROALIMENTACIÓN}

La retroalimentación positiva (amplificación) y negativo (amortiguamiento), son ingredientes claves de los sistemas complejos. El efecto de la acción de un agente es retroalimentado hacia el propio agente y por lo tanto, afecta la manera en que dicho agente se comportará en el futuro. Este conjunto de relaciones no lineales está en constante adaptación en contraposición al esquema lineal causa-efecto que predomina aún en el pensamiento de muchos profesionales.

En las interacciones entre los elementos de los sistemas complejos hay lazos de retroalimentación que aporta cada elemento y cada acción del sistema, los cuales son necesarios para el correcto funcionamiento o la autoorganización del sistema. En el manejo de la salud se debe incrementar la diversidad de conexiones del sistema, esto incrementa el flujo de información y promueve un adecuado funcionamiento, ya que hay más adaptabilidad creativa. ${ }^{20}$ 
La retroalimentación entre los síndromes geriátricos, tiene sus raíces en la pérdida de complejidad que el proceso de envejecimiento posee; complejidad implica armonía entre todos los sistemas del cuerpo, este proceso de debilidad senil disminuye la coordinación entre los sistemas, lo que atenúa la complejidad y hace más frágil a la persona. ${ }^{21}$

\section{EL SISTEMA TIENE UNA HISTORIA Y ES ÚNICO E IRREPETIBLE EN EL TIEMPO}

La historia del sistema es importante y no debe ignorarse. Incluso un cambio pequeño en determinadas circunstancias puede provocar grandes desviaciones en el futuro. La historia de un sistema complejo evoluciona de forma permanente, vinculado a su contexto y cambia si este es cambiado. Los sistemas interactúan y dejan informaciones que retroalimentan al mismo sistema o a otros subsistemas. En la atención médica de muchos países con intereses mayoritariamente comerciales de los servicios médicos, se prescriben antimicrobianos de última generación, que provocan desarrollo de resistencia a estos fármacos, de modo que la información inmunológica de una población y la resistencia de los agentes patógenos, son elementos que están cambiando la historia del sistema.

El cambio climático dado por la variabilidad natural del clima y por la actividad humana que altera la composición de la atmósfera mundial, refleja también la historia de un sistema, que está cambiando desfavorablemente e influyendo negativamente en varios aspectos de salud.

Cada individuo tiene particularidades diferentes que se deben tener en cuenta a la hora de un examen médico, tales como los umbrales del dolor, los sistemas inmunológicos, el contexto social, familiar, étnico y otros. Este nuevo enfoque, propone estudiar los problemas de la salud y la enfermedad bajo los principios y preceptos del pensamiento de la complejidad de modo que esto constituye un nuevo paradigma para las ciencias médicas y de la salud a nivel mundial.

Los profesionales de la salud al conservar adecuadamente y de forma sistemática las bases de datos formadas por las informaciones de su trabajo local, que muestran dinámicas e interacciones de sistemas, contribuyen a conservar información de la historia del sistema en que trabajan, que le sirven para realizar investigaciones con el propósito de resolver problemas locales. Estas bases de datos han servido para estudiar las dispersiones de epidemias de épocas pasadas y apoyan la actual toma de decisiones. ${ }^{22}$

\section{AUTOORGANIZACIÓN}

La autoorganización es la cualidad de ciertos sistemas de organizarse a sí mismos. Tendencia constante y espontánea de un sistema para generar patrones de comportamiento global, a partir de las interacciones entre sus componentes y a partir de las interacciones de estos con su entorno.

La autoorganización de sistemas puede ser una explicación alternativa para los ciclos de enfermedades que dependen de perturbaciones tales como la variabilidad climática; y de fluctuaciones de dinámica no lineal, que depende de retroalimentación del sistema, tales como interacción entre especies y la dependencia a la densidad poblacional, todos estos factores provocan oscilaciones en los sistemas. Los picos ínter epidémicos de enfermedades se deben a diversos tipos de interacciones, que pueden estar relacionadas con la inmunidad natural de 
una generación o de una comunidad o región; cuando la inmunidad decrece en una nueva generación o en una comunidad es entonces cuando se manifiestan las enfermedades. ${ }^{23}$

Los sistemas autoorganizados se mantienen dentro del estrecho dominio que oscila entre el orden inmutable y el desorden total, entre la constancia rígida y la turbulencia anárquica. Una condición muy especial, con suficiente orden para poder desarrollar procesos y evitar la extinción pero con una cierta dosis de desorden como para ser capaz de adaptarse a situaciones novedosas y evolucionar. Es lo que se conoce -desde antaño- como "transiciones de fase", o -más modernamente- el "borde del caos". Es en esta delgada franja en donde se ubican los fenómenos que edifican la vida y las sociedades.

Al comprender la consulta médica como un sistema complejo adaptativo, el médico cuenta con una valiosa herramienta teórica que le sirve de base para enfrentar la incertidumbre y lo impredecible, lo hace más capaz de enfrentar riesgos y le permite actuar con más creatividad. Existen modelos de rehabilitación mental basados en la autoorganización, ${ }^{24}$ otra aplicación se alcanza en el manejo de los dolores crónicos, lo que se logra cuando el paciente es tenido en cuenta, es escuchado y toma acción y responsabilidad en el enfoque terapéutico de su enfermedad. ${ }^{25}$ Otros autores refieren que el cerebro tiene sistemas caóticos adaptativos que le brindan la posibilidad potencial de acomodarse rápidamente a condiciones cambiantes, estos mecanismos han sido revelados mediante análisis no lineales [Faure P, Korn HC. R Acad Sci III. 2001;324(9):773-93].

Otros estudios informan que existen trastornos adaptativos con síntomas caóticos que no deben ser tratados de forma agresiva porque pueden empeorar la situación del paciente provocando trastornos crónicos. ${ }^{26}$

Finalmente se puede concluir que el sello distintivo de los sistemas complejos es su comportamiento de interacción no lineal e impredecible, estructurados con un gran número de elementos interactuando entre sí, cuyas repetidas interacciones resultan en un comportamiento colectivo que retroalimenta el comportamiento de las partes individuales. Involucran muchas conexiones entre individuos, estados y procesos. Con esta visión las organizaciones de atención de salud son sistemas sociales creados para organizar actividades y recursos necesarios para proveer este cuidado. Como seres vivos, los sistemas sociales son procesos interminables llenos de cambios los cuales crean nuevos órdenes a través del efecto de autoorganización.

Al conocer las propiedades de los sistemas complejos, el personal de la salud adquiere una visión holística y transdisciplinar de su trabajo, lo que motiva al paciente al incremento de su participación en el análisis y solución de sus problemas de salud. El médico general integral, en las redes locales, apoyado en este enfoque, es capaz de generar soluciones integradas a través de las conexiones históricas y sociales de cada localidad, además adquiere un conocimiento expandido, capacidad para el debate, discusión, adaptación a los cambios y desarrollo de nuevas metas y estrategias.

El estilo propuesto intenta movilizar la atención médica hacia atractores de salud utilizando cualquier elemento que pueda ayudar. La capacidad creativa de los estudiantes y profesionales se incrementa al hacerlos partícipes de proyectos de investigación para resolver problemas locales aumentando la educación con guías y simulaciones. Se aumenta el sentido de pertenencia a la comunidad al incentivar diferentes relaciones entre familia, ambiente, trabajo, escuela, entre otros. 
El pensamiento y las teorías de la complejidad privilegian la visón holista, transdisciplinar, el valor de la emergencia, la autoorganización, la conectividad, la retroalimentación, flexibilidad y la orientación al cambio, por lo que valora la importancia que tienen las pequeñas variaciones de las condiciones iniciales del entorno en la ocurrencia de fenómenos de gran alcance o viceversa; elementos que no deben ser desaprovechados en beneficio del mejoramiento de la visión integral y humanista de la atención médica y de los servicios de salud que caracteriza a la salud pública cubana.

\section{REFERENCIAS BIBLIOGRÁFICAS}

1. March D, Susser E. The eco-in eco-epidemiology. Inter J Epidemiol. 2006;35:1379-83.

2. Griffiths $F$, Byrne D. General practice and the new science emerging from the theories of chaos and complexity. Br J Gen Pract. 1998;48(435):1697-9.

3. Scott KJA. Dynamic Patterns. London, England: MIT Press, Cambridge, Mass; 1999.

4. Juarrero A. Dynamics in Action. London, England: MIT Press, Cambridge, Mass; 1999.

5. Salthe S. Development and Evolution: Complexity and Change in Biology. London, England: Cambridge, Mass, MIT Press;1996.

6. Van de Vijver, Salthe GS, Delpos M. Evolutionary Systems. Boston, London: Academic Publishes, Corcrecht; 1998.

7. De Landa M. A thousand years of non-linear history. New York: Swerve;2000.

8. García R. Sistemas Complejos. Barcelona: Editorial Gedisa;2006.

9. David TA, Liberatore V, Kurt P, Stange C, Aron D. Using Complexity Theory to build interventions that improve health care delivery in primary care. J Gen Intern Med. 2006;21:S30-4.

10. Leykum LK, Pugh J, Lawrence V, Parchman M, Noël PH, Cornell J, et al. Organizational interventions employing principles of complexity science have improved outcomes for patients with Type II diabetes. Implementation Science. 2007;2:28. [doi:10.1186/1748-5908-2-28].

11. Sturmberg JP. Systems and complexity thinking in general practice. Part 1clinical application. Australian Family Physician. 2007;36(3).

12. Love T, Dowell AC, Salmond C, Crampton P. Quality indicators and variation in primary care: modelling GP referral patterns. Family Prac. 2004;21:160-5.

13. Prieto Ramírez. La medicina desde la perspectiva del pensamiento de la complejidad. Rev Hum Med. 2003;3 (1). 
14. Frize M, Frasson C. Decision-support and intelligent tutoring systems in medical education. Clin Invest Med. 2000;23(4):266-9.

15. Eubank S, Guclu H, V Kumar A, Marathe MV, Srinivasan Aravind, Toroczkai Z, et al. Modeling disease outbreaks in realistic urban social networks.

Nature;2004;429(13).

16. Philippe $P$, Mansi $O$. Nonlinearity in the epidemiology of complex health and disease processes. Theoretical Medicine Bioethics.1998;19:591-607.

17. Betancourt JA, Brito HS, Ortiz EH, Rodríguez NS. Caracterización y análisis de series de tiempo de enfermedades respiratorias agudas en la provincia de Camagüey. Archivos Médicos Camagüey. En prensa 2008.

18. John RI, Innocent PR. Modeling uncertainty in clinical diagnosis using fuzzy logic IEEE. Trans Syst Man Cybern B Cybern. 2005;35(6):1340-50.

19. Massad E, Regina N, Ortega S, Struchinerc CJ, Nascimento MB. Fuzzy epidemics. Artificial Intelligence Med. 2003;29:241-59.

20. Holden LM. Complex adaptive systems: concept analysis. J Adv Nurs. 2005;52(6):651-7.

21. Musso CG, Núñez JF. Feed-back between geriatric syndromes: General System Theory in Geriatrics. Int Urol Nephrol. 2006;38(3-4):785-6.

22. Christakos G, Oleab RA, Yua HL. Recent results on the spatiotemporal modelling and comparative analysis of Black Death and bubonic plague epidemics. Public Health. 2007;121:700-20.

23. Diekmann O, J. Heesterbeek AP. Mathematical Epidemiology of Infectious Disease. New York: Wiley \& Sons;2000.

24. Lussi IA, Pereira MA, Pereira Jr. A. The proposal of Saracen's psychosocial rehabilitation: is it a model of self-organization? Rev Lat Am Enfermagem. $2006 ; 14(3): 448-56$.

25. Monsivais D. Self-organization in chronic pain: a concept analysis. Rehabil Nurs. 2005;30(4):147-51.

26. Golbin A, Umantsev A. Adaptive chaos: mild disorder may help contain major disease. Med Hypotheses. 2006;66(1):182-7.

Recibido: 3 de diciembre de 2008.

Aprobado: 23 de marzo de 2009. 
José Antonio Betancourt Betancourt. Centro de Medicina y Complejidad.

Universidad de Ciencias Médicas "Carlos J. Finlay". Apartado postal 370.Camagüey, Cuba.

E-mail: josebetancourt@infomed.sld.cu 